\title{
Relación entre el desarrollo neuromotor y el lenguaje oral en Educación Infantil
}

María Serna Carrión*

Máster Universitario en Neuropsicología y Educación, Universidad de La Rioja, Madrid, España.
*Correspondencia:

María Serna Carrión

maria.sernacarrion@educa.madrid.org

Citación:

Serna Carrión M. Relación entre el desarrollo neuromotor y el lenguaje oral en Educación Infantil.JONED. Journal of Neuroeducation. 2020; 1(1); $100-107$.

doi: 10.1344/joned.v1i1.31721

\section{Declaración ética}

El director responsable, supervisor y quien aprueba la investigación realizada durante el trabajo fin de máster es Héctor del Castillo Fernández (profesor titular e investigador en la UAH en el Departamento de Ciencias de la Educación).

\section{Conflicto de interés}

La autora, María Serna Carrión, declara la ausencia de conflicto de interés.

Editor:

Carmen Trinidad Cascudo

(Independiente)

Revisores:

Marcel Ruiz Mejías (Universitat Pompeu Fabra, Espanya) y Carmen Trinidad Cascudo (Independiente)

El manuscrito ha sido aceptado por todos los autores, en el caso de haber más de uno, y las figuras, tablas e imágenes no están sujetos a ningún tipo de Copyright.

\section{Resumen}

No cabe duda que el desarrollo neuromotor y la aparición del lenguaje son dos grandes acontecimientos de la infancia. En las últimas décadas, son muchos los autores interesados por analizar cómo la motricidad se relaciona con otros ámbitos del desarrollo, incluido el lenguaje, y encontramos estudios que evidencian la relación significativa entre ambas variables neuropsicológicas. El presente trabajo tiene como objetivo principal estudiar la relación entre el desarrollo neuromotor y el lenguaje oral en alumnos de la etapa de Infantil, con la finalidad de intentar aportar información que apoye estudios anteriores, impulsar la importancia que tiene la correcta estimulación de la motricidad y del lenguaje oral desde la infancia y, contribuir así a mejorar la acción educativa. Para ello, se realiza un estudio cuantitativo con un diseño no experimental, descriptivo y correlacional, con el que investigar esas dos variables a través de la aplicación de dos pruebas estandarizadas, la prueba de evaluación neuromotriz EVANM $^{1}$ y la prueba de lenguaje oral Navarra-Revisada PLON-R ${ }^{2}$, en una muestra compuesta por cuarenta niños de 5 y 6 años de edad (tercer curso de educación infantil) de un centro ordinario de la Comunidad de Madrid. Los resultados que se han obtenido del análisis estadístico realizado muestran que existe correlación positiva entre el tono muscular y la forma del lenguaje, lo que nos indica que para ciertos parámetros existe relación entre la neuromotricidad y el lenguaje. Además, teniendo en cuenta estos resultados, se propone un programa de intervención neuropsicológica con el objetivo de potenciar el adecuado desarrollo de dichas variables. Como conclusión, se destaca la importancia de conocer los procesos cerebrales de estas variables y su evaluación para, ofrecer una respuesta educativa más completa y realmente acorde al perfil del alumnado.

\begin{abstract}
There is no doubt that neuromotor development and the appearance of language are two great events of childhood. In recent decades, many authors have been interested in analyzing how motor skills relate to other areas of development, including language, and we have found studies that demonstrate the significant relation between both neuropsychological variables. The main objective of this project is to study the relation between neuromotor development and oral language in students in childhood education with the aim of providing information to support previous studies, to promote the importance of the correct motor and oral language stimulation from the childhood and thus improve the educational activity. For that purpose, a quantitative study is carried out with a non-experimental, descriptive and correlational design to study these two variables through
\end{abstract}


the application of two standardized tests, the EVANM neuromotive assessment test ${ }^{1}$ and the Navarra-Revised PLON-R oral language test ${ }^{2}$, with a sample including forty children between 5 and 6 year olds (third year of childhood education) from an ordinary school of the Community of Madrid. The results obtained from the statistical analysis carried out show that there is a positive correlation between the muscle tone and the language's form, which indicates that there is a relation between neuromotor skills and oral language for some parameters. In addition, taking into account these results, it is proposed a neuropsychological intervention program with the aim of promoting a correct development of these variables. As a conclusion, the importance of knowing the brain process of these variables and their evaluation is highlighted in order to offer a more complete educational response that is truly in line with the profile of the students.

Palabras clave: desarrollo neuromotor, lenguaje oral, infantil, correlación, intervención neuropsicológica.

\section{Introducción}

Afirma De Jager $^{3}$ que el desarrollo comienza, se alimenta y progresa con el movimiento que nutre cuerpo y mente, mejora logros y corrige fallos, y que a través del movimiento tiene lugar el desarrollo físico, el cual es esencial en la actividad cerebral y constituye el fundamento de las habilidades posteriores, tanto emocionales como sociales e intelectuales.

Por otra parte, Puyuelo et al. ${ }^{4}$ definen el lenguaje como una conducta comunicativa, una característica humana que realiza funciones esenciales a nivel cognitivo, social y comunicativo. Como afirma Bigas ${ }^{5}$, el desarrollo del lenguaje oral en la etapa de Educación Infantil tiene máxima importancia, ya que es el instrumento que permite al niño realizar un aprendizaje escolar satisfactorio, sobre el que se fundamentarán todos los conocimientos posteriores.

Diversos estudios de autores como Piaget $^{6}$ o Ferré7, demuestran la relación entre la motricidad, la cognición, y por tanto, el lenguaje oral, lo que incide directamente en el aprendizaje y en el rendimiento académico. Siguiendo a Velasco ${ }^{8}$, el desarrollo motor da lugar al lenguaje y posibilita el acceso a un mundo basado en la comunicación. Mediante la acción, el niño crea las bases para alcanzar el lenguaje $y$, posteriormente, los requisitos para que la acción se diferencie y mejore ${ }^{9}$. Además, diferentes autores afirman que algunas dificultades del lenguaje están asociadas, entre otras, a dificultades motoras ${ }^{10}$.

Teniendo en cuenta todo esto, desde la escuela, y ya desde las primeras etapas, es muy importante fomentar un adecuado desarrollo motor que permita al niño adquirir patrones y destrezas que vayan asentando las bases motrices y faciliten un mejor desarrollo del lenguaje, y en consecuencia, un mejor desarrollo integral y adaptación al entorno.

Este proyecto persigue estudiar la relación entre el desarrollo neuromotor y el lenguaje oral en niños del último curso de educación infantil, con la finalidad de aportar información que apoye estudios anteriores, fomentar la correcta estimulación de la motricidad y el lenguaje oral desde la etapa de Infantil y, contribuir así a mejorar la acción educativa en el aula y prevenir posibles dificultades que supongan problemas en el desarrollo. Por ello, se plantea la siguiente pregunta: ¿Qué relación hay entre el desarrollo neuromotor y el lenguaje oral en un grupo de niños de 5 y 6 años de edad de un centro ordinario de la Comunidad de Madrid? Para dar respuesta a esta pregunta, en este proyecto de investigación se describe un estudio cuantitativo con un diseño no experimental, descriptivo y correlacional, que estudia la relación de esas dos variables neuropsicológicas a través de la aplicación de dos pruebas estandarizadas, la prueba de evaluación neuromotriz EVANM y la prueba de lenguaje oral Navarra-Revisada, PLON-R, en una muestra de cuarenta niños de 5 y 6 años de edad que asisten a un centro ordinario de la Comunidad de Madrid para, posteriormente, analizar los resultados y elaborar una propuesta de intervención neuropsicológica adecuada. 


\section{Objetivos}

El objetivo general de la investigación se fundamenta en comprobar la relación entre el desarrollo neuromotor y el lenguaje oral en un grupo de cuarenta alumnos de tercer curso de educación infantil (5-6 años de edad) de un centro ordinario de la Comunidad de Madrid para, diseñar un programa de intervención para la estimulación de la motricidad y el lenguaje oral.

Para cumplir este objetivo general es necesario establecer unos objetivos específicos:

1. Evaluar el nivel del desarrollo neuromotriz en una muestra de alumnos de tercer curso de educación infantil (5-6 años de edad).

2. Evaluar el nivel de lenguaje oral en una muestra de alumnos de tercer curso de educación infantil (5-6 años de edad).

3. Estudiar la relación entre el desarrollo neuromotriz y el lenguaje oral en una muestra de alumnos de tercer curso de educación infantil (5-6 años de edad).

4. Diseñar un programa de intervención que estimule la motricidad y el lenguaje oral para una muestra de alumnos de tercer curso de educación infantil (5-6 años de edad) en base a los resultados obtenidos.

Teniendo en cuenta los objetivos establecidos, se plantean las siguientes hipótesis:

- Hipótesis 1: Se espera encontrar que los alumnos de 5 y 6 años no tengan unos patrones motrices plenamente desarrollados.

- Hipótesis 2: Se espera encontrar que los alumnos de 5 y 6 años no tengan un lenguaje oral plenamente desarrollado.

- Hipótesis 3: Se espera encontrar una relación positiva entre el desarrollo neuromotor y el desarrollo del lenguaje oral.

\section{Métodos}

La muestra que ha participado en esta investigación pertenece a la población de alumnos de un colegio ordinario y público de la Comunidad de Madrid. El centro está ubicado en el barrio de Delicias (distrito de Arganzuela), por lo cual las familias del centro presentan un nivel socioeconómico muy variado, pero, en general, el nivel es medio-alto, con estudios superiores en ambos progenitores y buena disposición para colaborar con el centro. Un $30 \%$ de las familias son inmigrantes, mayoritariamente de origen hispanoamericano, y en muchos de estos casos, los alumnos han nacido en España.

Dicha muestra se compone de cuarenta alumnos (veinte niños y veinte niñas) que están escolarizados en el centro desde los 3 años de edad, cursan tercero de educación infantil y tienen 5 años de edad cuando comienza el curso (septiembre 2017), pero en el momento de la aplicación de las pruebas (abril 2018), la edad oscila entre los 5-6 años de edad. El procedimiento de muestreo llevado a cabo es un método no probabilístico de carácter intencional y, los criterios de inclusión para la selección de la muestra han sido: no presentar necesidades educativas especiales o dificultades de aprendizaje, hablar y comprender correctamente el español y estar matriculado en tercer curso de educación infantil.

Para la obtención de la muestra, se contacta con varios centros de la Comunidad de Madrid. Finalmente, se selecciona aquel con mayor número de alumnos que podían participar en el estudio. Se realiza una reunión en el centro educativo para que conozcan la finalidad del estudio y la metodología de la investigación. Tras informar al centro y a las familias, se acepta la propuesta del proyecto y comienza la realización de las pruebas.

En la Tabla 1 se recogen las variables de estudio, los instrumentos aplicados y las mediciones recogidas para el análisis estadístico.

Tabla 1. Variables neuropsicológicas, instrumentos y medición

\begin{tabular}{|c|c|c|}
\hline Variable & Instrumento & Medición \\
\hline $\begin{array}{l}\text { Desarrollo } \\
\text { neuromotor }\end{array}$ & $\begin{array}{l}\text { Prueba de } \\
\text { evaluación } \\
\text { neuromotriz } \\
\text { EVANM }\end{array}$ & $\begin{array}{l}\text { Patrones motores, control postu- } \\
\text { ral y tono muscular: } \\
\text { - Cada apartado de } 1 \text { a } 5 \text { puntos, } \\
\text { se corresponde con un nivel de } \\
\text { adquisición. }\end{array}$ \\
\hline $\begin{array}{l}\text { Lenguaje } \\
\text { oral }\end{array}$ & $\begin{array}{c}\text { Prueba de } \\
\text { lenguaje oral } \\
\text { Navarra-Revisa- } \\
\text { da, PLON-R }\end{array}$ & $\begin{array}{l}\text { Forma, contenido y uso del len- } \\
\text { guaje: } \\
\text { - Cada subapartado: puntuación } \\
\text { entre } 0 \text { y } 2 \text { puntos. } \\
\text { - Cada apartado: cada puntuación } \\
\text { directa (PD) se corresponde con } \\
\text { una puntuación típica normaliza- } \\
\text { da S (PT). La suma de todas las } \\
\text { PD también se corresponde con } \\
\text { una PT, e indica el nivel de desa- } \\
\text { rrollo del lenguaje oral ("normal", } \\
\text { "necesita mejorar" o "retraso"). }\end{array}$ \\
\hline
\end{tabular}


La aplicación de las dos pruebas se lleva a cabo a lo largo de tres semanas, en horario escolar de mañana para evitar momentos de mayor fatiga y siempre en el mismo espacio (luminoso, tranquilo y silencioso), donde únicamente están presentes el evaluador y el evaluado. Ambas pruebas se realizaron de forma individual y coincidiendo con las sesiones de psicomotricidad para que todos los alumnos dispusiesen del material adecuado (calzado y ropa deportiva). Con cada alumno se realizó primero la prueba EVANM y seguidamente, la prueba PLON-R. La duración total para la realización de las dos pruebas fue aproximadamente de 15 minutos por cada niño.

Finalizada la evaluación, se organizan los datos de ambas pruebas en una base de datos y se realiza el análisis estadístico de los resultados. En primer lugar, los análisis descriptivos de la motricidad y lenguaje oral por separado y, posteriormente, el análisis correlacional entre las dos variables. Teniendo en cuenta la naturaleza ordinal de las dos escalas con las que se han medido las variables neuropsicológicas, para el análisis de los datos se ha utilizado el programa estadístico informático SPSS Statistics (versión 25) ya que cuenta con la prueba no paramétrica de Spearman, necesaria para llevar a cabo el análisis correlacional. Tras este análisis, se ha diseñado una intervención neuropsicológica adecuada a los resultados.

\section{Resultados}

\section{Análisis descriptivos}

Siguiendo el objetivo 1, "evaluar el nivel del desarrollo neuromotriz en una muestra de alumnos de tercer curso de educación infantil (5-6 años de edad)", se recogen en la Tabla 2 las frecuencias y los porcentajes para cada una de las habilidades motrices evaluadas y en la Figura 1 se representan las puntuaciones medias. Sobre estos resultados, cabe destacar que el gateo es el único patrón de motor automatizado, ya que la totalidad de la muestra ha obtenido la puntuación de "supera", lo que significa que se realiza con patrón cruzado y con agilidad. Por el contrario, los patrones de arrastre y triscado, aunque presentan puntuaciones muy variadas, son los que peores puntuaciones han obtenido. En cuanto al control postural, está adquirido por todos los alumnos; sin embargo, el tono muscular y los patrones motrices de la
Tabla 2. Frecuencias y porcentajes de los resultados del desarrollo neuromotor

\begin{tabular}{lcc}
\hline Frecuencia & $\begin{array}{c}\text { Porcentaje } \\
(\%)\end{array}$ & $\begin{array}{c}\text { Porcentaje } \\
\text { acumulado (\%) }\end{array}$ \\
\hline
\end{tabular}

\section{ARRASTRE}

\begin{tabular}{lccc}
\hline NO SUPERA SEVERO & 13 & 32.5 & 32.5 \\
\hline NO SUPERA ALTO & - & - & - \\
\hline NO SUPERA MEDIO & 7 & 17.5 & 50 \\
\hline NO SUPERA LEVE & 5 & 12.5 & 62.5 \\
\hline SUPERA & 15 & 37.5 & 100 \\
\hline
\end{tabular}

\section{GATEO}

\begin{tabular}{lccc}
\hline NO SUPERA SEVERO & - & - & - \\
\hline NO SUPERA ALTO & - & - & - \\
\hline NO SUPERA MEDIO & - & - & - \\
\hline NO SUPERA LEVE & - & - & - \\
\hline SUPERA & 40 & 100 & 100 \\
\hline
\end{tabular}

MARCHA

\begin{tabular}{lccc}
\hline NO SUPERA SEVERO & 3 & 7.5 & 7.5 \\
\hline NO SUPERA ALTO & - & - & - \\
\hline NO SUPERA MEDIO & - & - & - \\
\hline NO SUPERA LEVE & 5 & 12.5 & 20 \\
\hline SUPERA & 32 & 80 & 100 \\
\hline
\end{tabular}

TRISCADO

\begin{tabular}{lccc}
\hline NO SUPERA SEVERO & 12 & 30 & 30 \\
\hline NO SUPERA ALTO & 9 & 22.5 & 52.5 \\
\hline NO SUPERA MEDIO & 1 & 2.5 & 55 \\
\hline NO SUPERA LEVE & 10 & 25 & 80 \\
\hline SUPERA & 8 & 20 & 100 \\
\hline
\end{tabular}

\section{CARRERA}

\begin{tabular}{lccc}
\hline NO SUPERA SEVERO & 1 & 2.5 & 2.5 \\
\hline NO SUPERA ALTO & 1 & 2.5 & 5 \\
\hline NO SUPERA MEDIO & - & - & - \\
\hline NO SUPERA LEVE & 8 & 20 & 25 \\
\hline SUPERA & 30 & 75 & 100 \\
\hline
\end{tabular}

\section{TONO MUSCULAR}

\begin{tabular}{lccc}
\hline NO SUPERA SEVERO & - & - & - \\
\hline NO SUPERA ALTO & 1 & 2.5 & 2.5 \\
\hline NO SUPERA MEDIO & 8 & 20 & 22.5 \\
\hline NO SUPERA LEVE & 17 & 42.5 & 65 \\
\hline SUPERA & 14 & 35 & 100 \\
\hline
\end{tabular}

CONTROL POSTURAL

\begin{tabular}{lccc}
\hline NO SUPERA SEVERO & - & - & - \\
\hline NO SUPERA ALTO & - & - & - \\
\hline NO SUPERA MEDIO & - & - & - \\
\hline NO SUPERA LEVE & - & - & - \\
\hline SUPERA & 40 & 100 & 100
\end{tabular}


marcha y la carrera, aunque no se observa un gran porcentaje de alumnado con dificultades significativas, no están completamente superados.

Siguiendo el objetivo 2, "evaluar el nivel de lenguaje oral en una muestra de alumnos de tercer curso de educación infantil (5-6 años de edad)", se recogen en la Figura 2 las frecuencias de cada una de las dimensiones del lenguaje oral evaluadas. De estos resultados, hay que destacar que el contenido es el único ámbito con un desarrollo "normal" en la mayoría del alumnado; por el contrario, más de la mitad "necesita mejorar" en cuanto a la forma del lenguaje. Solo una minoría presenta "retraso" en dos dimensiones: la forma y el uso del lenguaje. Asimismo, en la Figura 3 observamos que la mayoría del alumnado presenta un nivel de lenguaje oral "normal"; sin embargo, solo un $10 \%$ se sitúa en la máxima puntuación. A pesar de que una parte de la muestra "necesita mejorar" diversos aspectos del lenguaje, ningún alumno ha presentado puntuaciones que se correspondan con un "retraso" en la puntuación total de la prueba; por ello, este valor no aparece en la figura.

\section{Análisis correlacional}

Siguiendo el objetivo 3, "estudiar la relación entre el desarrollo neuromotriz y el lenguaje oral en una muestra de alumnos de tercer curso de educación infantil (5-6 años de edad)", se ha realizado el análisis correlacional entre dichas variables, tal y como se observa en la Tabla 3. Podemos concluir que el gateo y el control postural son variables constantes (toda la muestra ha obtenido la misma puntuación) y no han podido ser susceptibles de correlación. Asimismo, cabe destacar que la única correlación significativa que se observa es entre el tono muscular y la forma, ya que el valor $p$ es ,014; por tanto, es menor que 0,05 con un coeficiente de correlación de rho $=$ ,386; luego, es una relación directa o positiva (cuanto mayor nivel de tono muscular, mayor nivel en la forma del lenguaje) y con una intensidad moderada. No se ha obtenido ninguna correlación significativa entre el resto de patrones motores y las diferentes dimensiones del lenguaje oral evaluadas porque los valores $p$ están por encima de 0,05.

\section{Discusión}

Tras analizar los datos, los resultados obtenidos indican, por un lado, que a nivel de desarrollo neuro-

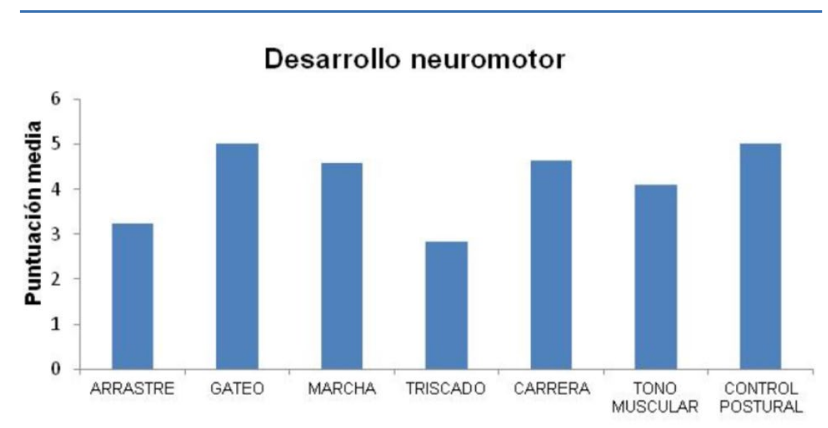

Figura 1. Medias de las habilidades motrices evaluadas

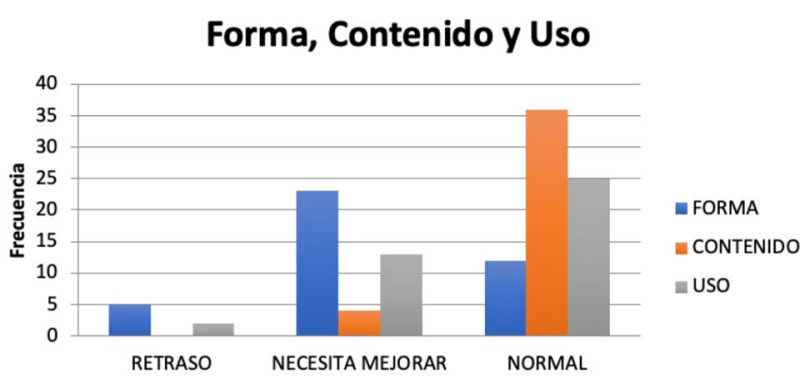

Figura 2. Frecuencia de los resultados de la forma, el contenido y el uso del lenguaje

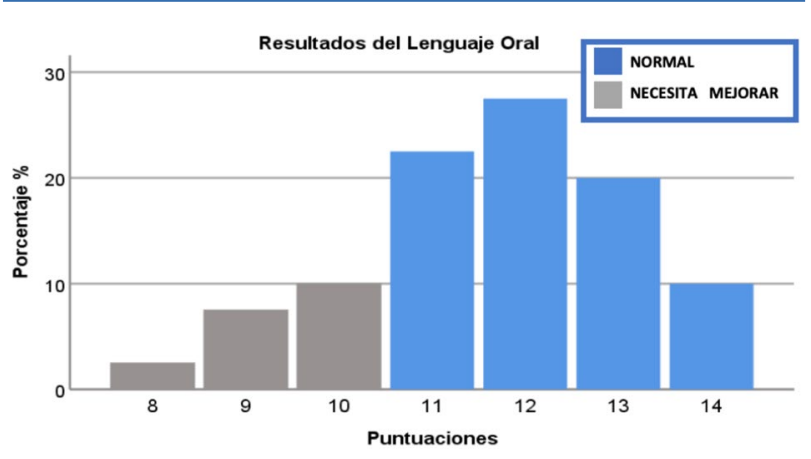

Figura 3. Porcentajes de los resultados obtenidos en el lenguaje oral

motor, los alumnos de la muestra no tienen todos los patrones motrices plenamente desarrollados, ya que solo se observa un patrón adquirido completamente por toda la muestra, el gateo, mientras que otros patrones como el arrastre (patrón previo al gateo) y el triscado no están superados en la mayoría del alumnado. En cuanto a la marcha y a la carrera, aunque no se observaron grandes dificultades durante la realización de los mismos, gran parte de la muestra no realizó estos patrones con total soltura y agilidad. Por lo tanto, se acepta la primera hipótesis establecida previamente: "Se espera encontrar que 
Tabla 3. Resultados del análisis de correlación

\begin{tabular}{|c|c|c|c|c|c|}
\hline & ARRASTRE & MARCHA & TRISCADO & CARRERA & TONO MUSCULAR \\
\hline \multicolumn{6}{|l|}{ FORMA } \\
\hline Coeficiente de correlación (rho) & ,163 &,- 027 &,- 221 & 184 &, $386(*)$ \\
\hline Sig. (bilateral) & ,314 & 870 & 170 & ,255 & 014 \\
\hline $\mathrm{N}$ & 40 & 40 & 40 & 40 & 40 \\
\hline \multicolumn{6}{|l|}{ CONTENIDO } \\
\hline Coeficiente de correlación (rho) & 016 &,- 012 & 073 &,- 124 &,- 052 \\
\hline Sig. (bilateral) & 923 & 942 & 656 & ,445 & ,751 \\
\hline $\mathrm{N}$ & 40 & 40 & 40 & 40 & 40 \\
\hline \multicolumn{6}{|l|}{ USO } \\
\hline Coeficiente de correlación (rho) &,- 030 &,- 083 &,- 061 &,- 097 &,- 004 \\
\hline Sig. (bilateral) &, 856 & 609 & ,707 &, 552 & 979 \\
\hline $\mathrm{N}$ & 40 & 40 & 40 & 40 & 40 \\
\hline
\end{tabular}

Nota: ${ }^{*} p<0,05$.

los alumnos de 5 y 6 años no tengan unos patrones motrices plenamente desarrollados".

Respecto al lenguaje oral, los resultados muestran en cada uno de los ámbitos evaluados (forma, contenido y uso) que los alumnos "necesitan mejorar" ciertos aspectos lingüísticos, ya que aún no están completamente adquiridos. Además, un pequeño porcentaje de la muestra presenta "retraso", es decir, mayores dificultades en la forma (pruebas de fonología, morfología y sintaxis) y el uso del lenguaje (pruebas de expresión verbal espontánea). De acuerdo con estos resultados, se cumple la hipótesis número dos de la investigación que es: "Se espera encontrar que los alumnos de 5 y 6 años no tengan un lenguaje oral plenamente desarrollado".

Por último, la hipótesis número tres, "Se espera encontrar una relación positiva entre el desarrollo neuromotor y el desarrollo del lenguaje oral", se acepta parcialmente, pues, tras el análisis correlacional de los datos, se constata que existe correlación positiva moderada únicamente entre el tono muscular y la forma del lenguaje, lo cual indica que solo para ciertos parámetros sí existe una relación significativa entre el desarrollo neuromotriz y el lenguaje.

Estos resultados apoyan en cierta medida los hallazgos de recientes investigaciones que tratan de encontrar evidencias en la relación entre estas dos variables.
Campo ${ }^{11}$ estudia la "importancia del desarrollo motor en la relación con los procesos evolutivos del lenguaje y la cognición en niños de 3 a 7 años de la ciudad de Barranquilla (Colombia)" utilizando como instrumento la prueba de desarrollo Battell ${ }^{12}$ y concluye que existe una relación positiva entre las tres variables. Ruiz et al. ${ }^{13}$ estudian: "Movimiento y lenguaje: Análisis de las relaciones entre el desarrollo motor y del lenguaje en la infancia" a través de la revisión de diversas investigaciones, y evidencian que hay más razones para considerar dichas relaciones que para rechazarlas. Libertus y Violi ${ }^{14}$ publican: "Sit to Talk: Relation between Motor Skills and Language Development in Infancy", una investigación que examina la relación entre las habilidades de alcanzar y sentarse, y el desarrollo posterior del lenguaje, con la que aportan resultados que muestran la relación entre la aparición de sentarse (entre los tres y los cinco meses de edad) y el posterior lenguaje receptivo (entre los diez y los catorce meses de edad) y afirman que las habilidades motoras desempeñan un papel crítico en el desarrollo temprano y en el aprendizaje.

También encontramos estudios donde la muestra presenta algún tipo de trastorno o síndrome específico como Michel et al. ${ }^{15}$, que realizan un estudio con niños de entre 5 y 7 años, algunos con problemas de coordinación motriz y otros sin problemas, y concluyen que los que presentaban estos problemas 
manifestaban dificultades en las funciones ejecutivas y en las destrezas académicas. Posteriormente, Avedaño et al. ${ }^{16}$ analizan la comorbilidad entre el trastorno específico del lenguaje (TEL) y los trastornos de las destrezas motoras y del desarrollo de la coordinación, para describir la relación entre el desarrollo motriz y lenguaje, y constatan que los niños con TEL presentan un nivel más bajo que sus iguales en tareas de motricidad, en el aprendizaje de símbolos grafomotrices y en habilidades de movimiento. Por último, Rodríguez et al. ${ }^{17}$ investigan la influencia de una intervención psicomotriz en el desarrollo del lenguaje en niños con síndrome de Down (de 4 y 5 años de edad); los resultados muestran que esta intervención tiene resultados positivos en la mejora del lenguaje comprensivo y expresivo y en la motricidad gruesa y fina.

Ruiz et al. ${ }^{13}$ afirman que la literatura científica es abundante y existen diversas líneas de investigación, pero, frente a posturas que creen en la independencia de los procesos de desarrollo, hay una tendencia a considerar que el desarrollo motor y el desarrollo del lenguaje mantienen una estrecha relación ${ }^{18-19}$.

\section{Conclusiones}

En definitiva y teniendo en cuenta todo lo anterior, las conclusiones de este estudio se concretan, por un lado, en el análisis estadístico realizado, que evidencia que los patrones básicos del movimiento y el lenguaje oral de la muestra compuesta por niños de 5-6 años de edad no están plenamente desarrollados. Además, los resultados de las pruebas aplicadas (EVANM y PLON-R) muestran que existe una relación significativa entre el tono muscular y la forma del lenguaje; en este sentido, para ciertos parámetros existe relación entre el desarrollo neuromotriz y el lenguaje oral.

Por otro lado, y siguiendo la revisión teórica llevada a cabo, se concluye que el desarrollo neuromotor y el lenguaje oral son fundamentales para el desarrollo integral del niño, por lo que su correcta estimulación ya desde las etapas tempranas incidirá directamente en los aprendizajes superiores futuros. Por ello, en el ámbito educativo se hace necesaria la evaluación neuropsicológica que permita elaborar y establecer programas de intervención que estimulen de forma adecuada variables como la motricidad y el lenguaje oral en la escuela. Por lo tanto, para poder ofrecer una respuesta educativa realmente acorde al perfil del alumnado, que respete los tiempos del desarrollo y las características individuales, es necesario que la formación de los profesionales de la enseñanza incluya el estudio de los procesos cerebrales, ya que ello les permitirá conocer las bases neuropsicológicas de estas u otras variables.

\section{Limitaciones}

La presente investigación muestra una serie de limitaciones que deberán tenerse en cuenta en futuras investigaciones.

En primer lugar, la totalidad de la muestra está compuesta únicamente por alumnado del tercer curso de educación infantil, por lo que los resultados obtenidos en este estudio no pueden generalizarse a otros niveles. Asimismo, surge la limitación del tiempo para realizar la evaluación, y es que, aunque las dos pruebas utilizadas son adecuadas y se han recogido todos los datos correctamente, ampliar este tiempo de evaluación supondría aplicar las pruebas con mayor tranquilidad y rigurosidad, así como poder observar más tiempo a los sujetos y recabar más información. Por último, surge también la limitación en el tiempo para poder llevar a cabo el programa de intervención y analizar el impacto que tiene su aplicación en la muestra.

A pesar de estas limitaciones, los datos aportados en este estudio resultan relevantes, ya que aportan un análisis completo de la relación entre el desarrollo neuromotor y el lenguaje oral en alumnado de tercer curso de educación infantil y ponen de manifiesto la importancia que tiene en esta etapa una adecuada estimulación de la motricidad y el lenguaje para el desarrollo integral del niño y la adquisición de aprendizajes.

\section{Agradecimientos}

A todos los miembros de la comunidad educativa que colaboraron en este proyecto y a Eduardo Pardo San Llorente por su apoyo y aporte tecnológico. 


\section{Referencias}

1. Díaz-Jara M, Martín-Lobo P, Vergara-Moragues E, Navarro-Asensio E, Santiago-Ramajo S. Prueba de evaluación neuromotriz (EVANM). La Rioja: UNIR; 2015.

2. Aguinaga $G$, Armentia $M$, Fraile $A$, Olangua $P$, Uriz N. Prueba del lenguaje oral Navarra-revisada, PLON-R. Madrid: TEA; 2005.

3. De Jager M. Mente en Acción. Movimientos que mejoran la mente. Jaén: Tucco Publishin, S. L; 2010.

4. Puyuelo M, Wiig E, Renom J, Solanas, A. Batería de lenguaje objetiva y criterial. BLOC. Barcelona: Masson; 1998.

5. Bigas M. La importancia del lenguaje oral en educación infantil. Aula de Innovación Educativa [Internet]. 1996 [consultado en marzo de 2018]; 46. Disponible en: https://logopedicum. com/wp-content/uploads/2017/02/la-importancia-del-lenguaje-oral-en-educacion-infantil.pdf

6. Piaget J. Epistemología y Psicología de la Identidad. Buenos Aires: Paidós; 1971.

7. Ferré J, Ferré M. Neuropsicopedagogía infantil. Bases neurofuncionales del aprendizaje cognitivo y emocional. Barcelona: Lebón; 2013.

8. Velasco K. El lenguaje oral y el movimiento en estrecha comunicación. Espacio Logopédico [Internet]. 2014 [consultado en marzo de 2018]; Disponible en: https://www.espaciologopedico.com/revista/articulo/332/el-lenguaje-oral-y-el-movimiento-en-estrecha-comunicacion.html

9. Tejedor MC, Pérez MM. La evolución del lenguaje y la motricidad y la conexión entre ellas en el niño de 0 a 6 años. Comunicación presentada en el Congreso de Córdoba; 1997 dic; Córdoba, España.

10. Conde-Gunzón PA, Conde-Gunzón MJ, Bartolomé-Albistegui MT, Quirós-Expósito P. Perfiles neuropsicológicos asociados a los problemas del lenguaje oral infantil. Rev Neurol [Internet]. 2009 [consultado en marzo de 2018]; 48(1); 32-38. Disponible en: https://www.neurologia.com/ articulo/2008164

11. Campo LA. Importancia del desarrollo motor en relación con los procesos evolutivos del lenguaje y la cognición en niños de 3 a 7 años de la ciudad de Barranquilla (Colombia). Salud Uninorte [Internet]. 2010 [consultado en marzo de 2018]; 26 (1); 65-76. Disponible en: http://www.scielo.org.co/pdf/sun/ v26n1/v26n1a08.pdf

12. Newborg J, Stock J, Wnek L. Battelle Inventario de Desarrollo.
Manual de aplicación. $2^{\mathrm{a}}$ ed. Madrid: Publicaciones de Psicología aplicada TEA; 1998.

13. Ruiz LM, Ruiz A, Linaza JL. Movimiento y lenguaje: Análisis de las relaciones entre el desarrollo motor y del lenguaje en la infancia. Rev Int Cienc Deporte [Internet]. 2016 [consultado en marzo 2018]; 12 (46); 382-397. Disponible en: https:// dialnet.unirioja.es/servlet/articulo?codigo $=5739836$

14. Libertus K, Violi DA. Sit to Talk: Relation between Motor Skills and Language Development in Infancy. Front Psychol [Internet]. 2016 [consultado en marzo 2018]; 7; 475. Disponible en: https://www.frontiersin.org/articles/10.3389/ fpsyg.2016.00475/full

15. Michel E, Roethlisberger M, Neuenschwander R, Roebers CM. Development of Cognitive Skills in Children with Motor Coordination Impairments at 12-Month Follow-up. Child Neuropsychology [Internet]. 2011 [consultado en marzo 2018]; 17; 151-172. Disponible en: https://www.tandfonline.com/doi/ab s/10.1080/09297049.2010.525501

16. Avedaño $P$, Hernández $C$, Fernandes $S$, Caniuqueo $A$, Fernandes J, Pérez J. Trastorno específico del lenguaje y de las destrezas motoras. Una revisión bibliométrica. ARETÉ [Internet]. 2015 [consultado en marzo 2018]; 15(1); 89-98. Disponible en: http://revistas.iberoamericana.edu.co/index.php/arete/ article/view/927 (2015).

17. Rodríguez MT, Gómez IM, Prieto-Ayuso A, Gil-Madrona P. La educación psicomotriz en su contribución al desarrollo del lenguaje en niños que presentan necesidades específicas de apoyo educativo. Revista de Investigación en Logopedia [Internet]. 2017 [consultado en marzo 2018]; 7(1); 89-106. Disponible en: http://revistalogopedia.uclm.es/ojs/index.php/ revista/article/view/175

18. Wang MV, Lekhal R, Aaro LE, Holte A, Schjolberg S. The developmental relationship between language and motor performance from 3 to 5 years of age: A prospective longitudinal population study. BMC Psychol [Internet]. 2014 [consultado en marzo 2018]; 2; 34. Disponible en: http://www.biomedcentral.com/2050-7283/2/34

19. Iverson JM. Developing language in a developing body: the relationship between motor development and language development. J Child Lang [Internet]. 2010 [consultado en marzo 2018]; 37; 229-261. Disponible en: https://www.ncbi.nlm.nih. gov/pmc/articles/PMC2833284/ 